\title{
Tracing the steps of Zika virus
}

Epidemiological data have linked the infection of pregnant women with Zika virus (ZIKV) to microcephaly of their babies, and ZIKV was detected in brain tissue and amniotic fluid of microcephalic fetuses; however, direct experimental evidence of a causal link was scarce. Now, for the first time, three studies in pregnant mice show pathological changes in unborn mice following infection with ZIKV.

Immunocompetent mice show no clinical signs of disease following infection with ZIKV. However, it has been shown previously that ZIKV infection of adult mice in which the receptor for type I interferon (IFNAR) had been knocked out or of mice that were treated with an IFNAR-blocking antibody causes disease. Now, Mysorekar, Diamond and colleagues have developed two fetal infection models. In one model,

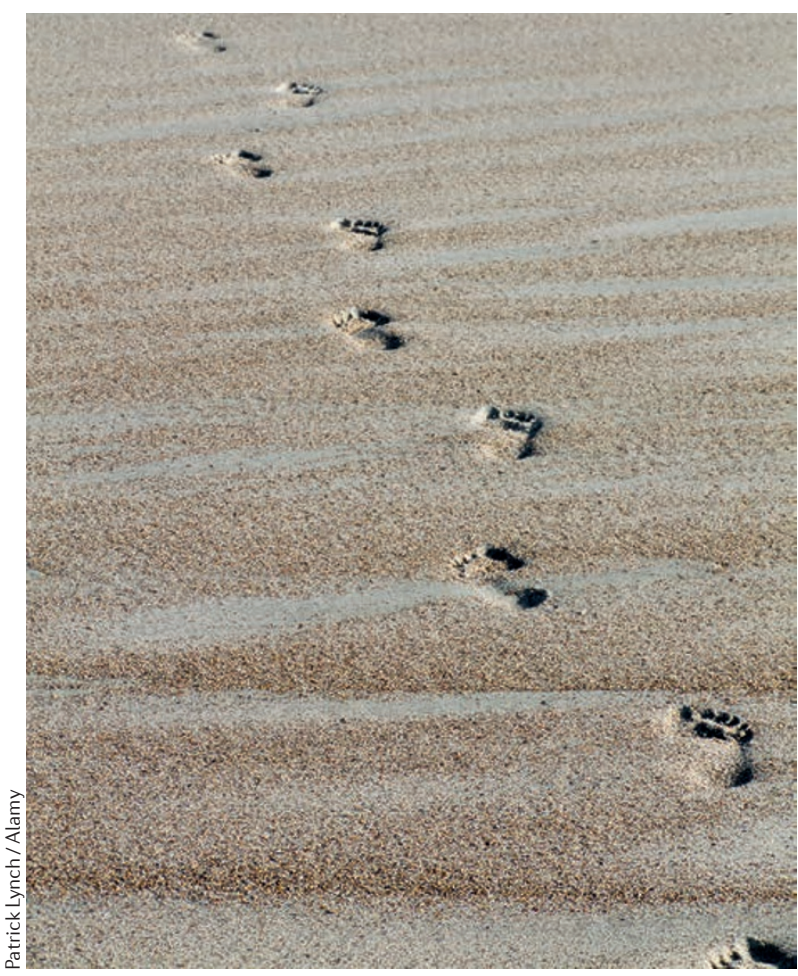

Ifnar ${ }^{-1-}$ female mice were mated with wild-type males; in the other, female and male wild-type mice were mated and the pregnant mice were treated with an anti-IFNAR antibody. In both models, the pregnant mice were challenged subcutaneously with ZIKV 1 week after conception and fetuses were analysed a week later, when they had completed approximately two-thirds of intrauterine development. Although most of the Ifnar ${ }^{+/-}$fetuses had died, the surviving fetuses showed growth retardation. Microcephaly was not observed, but the authors speculated that this might be due to the timing of infection and of brain development, a large part of which only happens after birth in mice. The fetuses from the antibody-treated mice also showed growth retardation but normal survival. In both models, ZIKV was detected by RT-PCR in placental and fetal tissue with high levels of virus in the fetal head. Furthermore, the placenta showed areas of necrosis, microscopic signs of apoptosis and vascular damage, and ZIKV was detectable by immunofluorescence and electron microscopy in placental cells. ZIKV was undetectable by immunofluorescence in fetal brains, but the hindbrain and the midbrain showed high levels of apoptosis. Taken together, these results show that ZIKV infects the placenta, is transferred to the fetus and compromises fetal development.

Similarly, Peron, Muotri, Beltrão-Braga and colleagues also detected growth retardation and high ZIKV loads in the brains of mice that were infected in utero. In contrast to the study above, these authors used SIL mice, a mouse strain that has immune system abnormalities and is often used to study autoimmune and neurological diseases. Pregnant
SJL mice were infected intravenously with ZIKV 10-13 days after conception and the newborn pups were analysed. Interestingly, in this model, brain abnormalities, specifically thinning of the cortex and reduced cell numbers, could be observed.

A third study by $\mathrm{Xu}$, Qin and colleagues investigated fetal brain pathology by injecting ZIKV directly into the lateral ventricle of 13.5-dayold fetuses in utero. The authors detected virus replication 3-5 days later by RT-PCR and by immunofluorescence. Infected cells were most abundant in ventricular and subventricular zones, where many neural precursor cells are located. Infected fetuses had smaller brains, a thinner cortical layer and enlarged ventricles compared with control fetuses 5 days after challenge. By co-staining for ZIKV and different cellular markers, the authors demonstrated that neural progenitor cells are the main target of ZIKV, although other neural cells were infected as well, and infection inhibits cellular proliferation and differentiation. Transcriptome analysis showed a downregulation of genes that are involved in cell and organ development and an upregulation of genes that are involved in immune responses and apoptosis.

Together, the three studies provide strong experimental support for the pathogenic potential of ZIKV during fetal development. By showing that, in mice, ZIKV infects the placenta and the fetal brain, where it induces neuronal apoptosis, they present a causal link between ZIKV infection and microcephaly.

Ursula Hofer

ORIGINAL ARTICLES Miner, J. J., Cao, B., Govero, J. et al. Zika virus infection during pregnancy in mice causes placental damage and fetal demise. Cell http://dx.doi.org/10.1016/j. cell.2016.05.008 (2016)|Cugola, F. R., Fernandes, I. R., Russo, F. B. et al. The Brazilian Zika virus strain causes birth defects in experimental models. Nature http://dx.doi.org/10.1038/nature18296 (2016) | Li, C., Xu, D., Hong, S. et al. Zika virus disrupts neural progenitor development and leads to microcephaly in mice. Cell Stem Cell http:// dx.doi.org/10.1016/j.stem.2016.04.017 (2016) 